\title{
On the Pascal Hexagram.
}

By Prof. J. JACK.

\$1. Proof in case of the circle.

Figure 9.

ABCDEF is any cyclic hexagon;

$A B, D E$ meet in $G$, $\mathrm{BC}, \mathrm{EF}, " \mathrm{H}$, $\mathrm{CD}, \mathrm{FA}, ", \mathrm{~K}$; then $\mathrm{G}, \mathrm{H}, \mathrm{K}$ are in a straight line.

Draw KLNM parallel to $\mathrm{BC}$ and produce DE, EF, $\mathrm{AB}$ to meet it in $I_{4}, N, M$. Join DA, DM and let BC, DE meet in $P$.

$\angle \mathrm{PCD}=\angle \mathrm{BAD} \quad \because \mathrm{ABCD}$ is a cyclic quadrilateral, and $\angle \mathrm{PCD}=\angle \mathrm{DKM} \quad \because \mathrm{KM}$ is parallel to $\mathrm{CB}$;

$\therefore \quad \angle \mathrm{BAD}=\angle \mathrm{DKN}$; $\therefore \mathrm{DAMK}$ is a cyclic quadrilateral.

Again $\angle D E H=\angle D A K \quad \because A F E D$ is cyclic ;

and $\angle \mathrm{DMN}$, i.e., $\angle \mathrm{DMK}=\angle \mathrm{DAK} \because \mathrm{DAMK}$ is cyclic;

$\therefore \angle \mathrm{DEH}=\angle \mathrm{DMN}$ and $\therefore \mathrm{DMNE}$ is cyclic.

$$
\begin{aligned}
& \text { Again } \frac{\mathrm{PB}}{\mathrm{PE}}=\frac{\mathrm{PD}}{\mathrm{PC}}=\frac{\mathrm{DL}}{\mathrm{LK}} \\
& \text { and } \frac{\mathrm{PE}}{\mathrm{PH}}=\frac{\mathrm{LE}}{\mathrm{LN}}=\frac{\mathrm{LM}}{\mathrm{LD}} ; \therefore \frac{\mathrm{PB}}{\mathrm{PH}}=\frac{\mathrm{LM}}{\mathrm{LK}} \text {; } \\
& \therefore G, H, K \text { are in a straight line. }
\end{aligned}
$$

\section{Figures 10 and 11.}

$\$ 2$. $O$ is any point on a chord $A B$ of a conic; the focus $S$, the directrix and $e$ are given; the eccentric circle of $O$ is described. Through $\mathrm{O}$, radii $\mathrm{O} a, \mathrm{O} b$ are drawn parallel to $\mathrm{SA}, \mathrm{SB}$, in opposite 
sense when O, S are on the same side of the directrix (Fig. 10) and in the same sense when on opposite sides (Fig. 11).

Then $a b$ passes through $\mathbf{S}$.

For

$$
\frac{\mathrm{O} a}{\mathrm{OK} \sin \theta}=\frac{\mathrm{SA}}{\mathrm{AK} \sin \theta} \text {; }
$$

$\therefore \mathrm{S}, a, \mathbf{K}$ are in a straight line. So $\mathrm{S}, b, \mathrm{~K}$ are in a straight line; $\therefore a b$ goes through $\mathrm{S}$.

\section{§3. Pascal's Theorem for the Conic (generally).}

$A B C D E F$ is any hexagon inscribed in a conic.

$\mathrm{AB}, \mathrm{DE}$ meet in $\mathrm{O}_{1} ; \mathrm{BC}, \mathrm{EF}$ meet in $\mathrm{O}_{2}$, and $\mathrm{CD}, \mathrm{FA}$ meet in $\mathrm{O}_{3}$.

Then $\mathrm{O}_{1}, \mathrm{O}_{2}, \mathrm{O}_{3}$ are in a straight line.

The focus $\mathrm{S}$, the directrix and $e$ are given.

Draw the eccentric circles of the points $\mathrm{O}_{1}, \mathrm{O}_{3}, \mathrm{O}_{3}$ and draw in each of the circles the six radii parallel to SA, SB, SC, SD, SE and SF, in the opposite sense when $O$ and $S$ are on the sarne side of the directrix and in the same sense when $O, S$ are on opposite sides of the directrix.

Let the radii be $\mathrm{O}_{1} a_{1}, \mathrm{O}_{1} b_{1}$, etc.; $\mathrm{O}_{2} a_{2}, \mathrm{O}_{2} b_{2}$, etc., etc.

Join the points $a_{1} b_{1}, b_{1} c_{1}, c_{1} d_{1}, d_{1} e_{1}, e_{1} f_{1}, f_{1} a_{1}$;

$$
\begin{array}{lll}
a_{2} b_{2}, & b_{2} c_{2}, & c_{2} d_{2}, \text { etc., } \\
a_{3} b_{3}, & b_{3} c_{3}, & c_{3} d_{3}, \text { etc. }
\end{array}
$$

Then the three cyclic hexagons $(a b c d e f)_{1},(a b c d e f)_{2},(a b c d e f)_{3}$ are similar and similarly situated.

Let $a_{1} b_{1}, d_{1} e_{1}$ meet in $g_{1}$,

$$
b_{1} c_{1}, e_{1} f_{1} \quad ", \quad h_{1},
$$

and $c_{1} d_{1}, f_{1} a_{3}, ", k_{1}$,

and similarly for the other two hexagons let the corresponding sides meet in $g_{2}, h_{2}, k_{7}$ and $g_{3}, h_{3}, k_{3}$.

Then $g_{2} h_{3} k_{1}, g_{2} h_{2} k_{2}, g_{3} h_{3} k_{3}$ are straight lines by the proof of the theorem in the case of a circle. 
Now from the nature of the eccentric circle, $a_{1} b_{1}, d_{1} e_{1}$ meet in $\mathrm{S}$, that is, the point $g_{1}$ is $\mathrm{S}$.

Similarly $\quad " \quad, \quad h_{2}$ is $\mathbf{S}$

and $\quad, \quad k_{3}$ is $\mathrm{S}$.

Hence the three straight lines, $g_{1} h_{1} k_{1}, g_{2} h_{2} k_{2}, g_{3} h_{5} k_{3}$ have one point $\mathbf{S}$ common and they are parallel, because the figures are similar and similarly situated;

$\therefore$ the three lines are coincident.

Now taking the three triangles $\mathrm{O}_{1} a_{1} g_{1}, \mathrm{O}_{2} a_{2} g_{2}, \mathrm{O}_{3} a_{3} g_{3}$ which are similar, we have $\quad \frac{\mathrm{O}_{1} g_{1}}{\mathrm{O}_{1} a_{1}}=\frac{\mathrm{O}_{2} g_{2}}{\mathrm{O}_{2} a_{2}}=\frac{\mathrm{O}_{2} g_{3}}{\mathrm{O}_{3} a_{3}}$

and if $\mathrm{O}_{1} m_{1}, \mathrm{O}_{2} m_{2}, \mathrm{O}_{3} m_{3}$ are the perpendiculars from $\mathrm{O}_{1}, \mathrm{O}_{2}, \mathrm{O}_{3}$ to the directrix, we have

it therefore follows that

$$
\frac{\mathrm{O}_{1} a_{1}}{\mathrm{O}_{1} m_{1}}=\frac{\mathrm{O}_{2} a_{2}}{\mathrm{O}_{2} m_{2}}=\frac{\mathrm{O}_{3} a_{3}}{\mathrm{O}_{3} m_{3}}=e ;
$$

$$
\frac{\mathrm{O}_{1} g_{1}}{\mathrm{O}_{1} m_{1}}=\frac{\mathrm{O}_{2} g_{2}}{\mathrm{O}_{2} m_{2}}=\frac{\mathrm{O}_{3} g_{3}}{\mathrm{O}_{3} m_{3}}
$$

$\therefore \mathrm{O}_{1}, \mathrm{O}_{2}, \mathrm{O}_{3}$ are in a straight line, and it passes through the point in which the Pascal line of the cyclic hexagons meets the directrix.

On Newton's Theorem in the Calculus of Variations. By J. H. Maclagan-Wedderburn, M.A. 